\title{
FUERZA DE LOS ÁCIDOS Y DE LAS BASES Y CRITERIOS DE CÁlCULO DEL PH
}

\author{
BORSESE, A. \\ Istituto di Chimica Generale. Viale Benedetto XV, 3. 16132 Genova. Italia.
}

\section{SUMMARY}

The author establishes a classification of acids and bases depending on their force, based on quantitative criteria from the percentage of chemical matter intervening in a reaction. Thence he analyzes the consequences for the value of the equilibrium constants and studies different cases.

\section{INTRODUCCIÓN}

Durante mucho tiempo, el lenguaje ha tenido en Química un significado cualitativo que no correspondía a las exigencias de rigurosidad y de precisión que el conocimiento científico requiere actualmente.

Los ácidos y las bases, por ejemplo, eran clasificados en dos categorías: fuertes y débiles. Más tarde, dado el insuficiente poder designativo de estos dos adjetivos, se introdujeron otros como "fuertísimo", "semifuerte", "semidébil", "debilísimo", etc. Se trataba de un criterio de clasificación completamente inadecuado. En efecto, muy probablemente todos ios adjetivos del diccionario no habrían bastado para distinguir entre sí todos los ácidos y todas las bases.

El gran desarrollo de la Quimica Física ha permitido más tarde "medir" la intensidad de los comportamientos enriqueciendo sensiblemente el lenguaje químico y dándole una exacta connotación científica.

Pauling (1956 y 1976), por ejemplo, ha calculado varias veces la fuerza de los ácidos en solución acuosa utilizando datos termodinámicos.

La clasificación de los ácidos y de las bases se realiza actualmente sobre la base de los valores numéricos de las constantes de equilibrio.

El lenguaje de las palabras para indicar "el grado" de una cierta propiedad tiene enormes límites $y$, sobre todo, a nivel didáctico, debería utilizarse lo menos posible.
Por otra parte, una clasificación de los ácidos y de las bases fundada sobre los valores de las constantes de equilibrio parece permitir una valoración cuantitativa de la contribución ácida o básica sin tener que memorizar previamente si aquel ácido o aquella base son fuertes o débiles. No se propone aquí que se abandone el concepto de "fuerza" de los ácidos y de las bases, sólo se considera más racional deducir "la fuerza" a partir del valor tabulado de las constantes de equilibrio que representan el comportamiento ácido o básico de una cierta especie química.

Sin embargo, es necesario preguntarse a este propósito: ies suficiente conocer el valor de la constante de equilibrio para deducir la situación de un proceso y para afirmar si un ácido o una base han reaccionado mucho o poco? La respuesta es no.

Para poder afirmar si un ácido o una base son fuertes o débiles también es necesario tomar en consideración la concentración de la solución.

Consideremos, a modo de ejemplo, el ácido acético que tiene una $\mathrm{K}_{\mathrm{a}} \approx 10^{-5}$ y que tanto manuales escolares como profesores clasifican entre los ácidos débiles.

Si expresamos $K_{a}$ en función del grado de disociación, obtenemos, como ya se sabe, la siguiente fórmula:

$$
\mathrm{K}_{\mathrm{a}}=\frac{\alpha^{2}}{1-\alpha} . \mathrm{c}
$$


que precisamente nos indica que el carácter de la reacción expresada por $\alpha$ depende tanto de $\mathrm{K}_{\mathrm{a}}$ como de $\mathrm{c}$.

Será la relación $\mathrm{K}_{\mathrm{a}} / \mathrm{c}$ lo que nos permitirá poder afirmar si nuestro ácido acético se comportará como un ácido fuerte o como un ácido débil. Es decir, en general, para deducir la fuerza de un ácido o de una base, habrá que considerar la relación $\mathrm{K}_{\mathrm{a}} / \mathrm{c}$ o la $\mathrm{K}_{\mathrm{b}} / \mathrm{c}$ respectivamente.

Pero, ¿cómo establecer el valor de estas relaciones por las cuales un ácido o una base se pueden considerar fuertes y el valor de estas relaciones por las que un ácido y una base se pueden considerar débiles?

Tenemos que decidir a partir de qué valor del grado de disociación consideramos un ácido o una base fuertes y hasta qué valor del grado de disociación consideramos un ácido o una base débil.

La decisión adoptada, que consiste en considerar fuertes un ácido y una base de los que reacciona más del $95 \%$, y débiles un áciđo y una base de los que reacciona menos del $5 \%$, se justifica principalmente por las siguientes razones: el $\mathrm{pH}$ es medido con una imprecisión de 0,02 unidades de $\mathrm{pH}$, que corresponden a una diferencia del $5 \%$ entre los valores de la concentración de $\mathrm{H}+$; y las $\mathrm{K}_{\mathrm{a}}$ y las $\mathrm{K}_{b}$ se calcula con una imprecisión cuya media es del $5 \%$.

Ahora ya tenemos los datos necesarios para calcular los valores de las dos relaciones $\mathrm{K} / \mathrm{c}$ que nos permitirán clasificar los ácidos y las bases.

De hecho, tendremos:

$\mathrm{K} / \mathrm{c}=(0,95)^{2} /(1 \cdot 0,95)=18,05$

$K / c=(0,05)^{2} /(1-0,05)=2,6 \times 10^{-3}$

Es decir, consideraremos fuertes los ácidos y las bases para los que $\mathrm{K}_{(\mathrm{a}} \mathrm{O}_{b)} / \mathrm{c}$ será mayor que 18,05 , y débiles los ácidos y las bases para los cuales esta relación será menor que $2,6 \times 10^{-3}$.

Los ácidos y las bases para los cuales $\mathrm{K}_{(\mathrm{a}} \mathrm{O}_{\mathrm{b})} / \mathrm{C}$ estará comprendido entre $2,6 \times 10^{-3}$ y 18,05 no podrán ser considerados fuertes ni débiles en el sentido de que no podremos, en los balances de masa del ácido y de la base, descuidar ninguna de las dos formas (nos referimos a los ácidos y a las bases monopróticos) presentes en la solución; los llamaremos ácidos y bases medios o "intermedios".

Veamos ahora cómo se puede calcular el pH de un ácido o de una base monopróticos según sus "fuerzas". Como ya se sabe, las dos ecuaciones que representan los dos procesos son las siguientes:

$$
\begin{aligned}
& \mathrm{HA}+\mathrm{H}_{2} \mathrm{O} \rightleftarrows \mathrm{A}^{-}+\mathrm{H}_{3} \mathrm{O}^{+} \\
& \mathrm{B}+\mathrm{H}_{2} \mathrm{O} \rightleftarrows \mathrm{BH}^{+}+-\mathrm{OH}
\end{aligned}
$$

También está la contribución debida a la disociación del agua:

$$
2 \mathrm{H}_{2} \mathrm{O} \rightleftarrows \mathrm{H}_{3} \mathrm{O}^{+}+-\mathrm{OH}
$$

Así las relaciones que existen entre las concentraciones de las diferentes especies en los dos casos son:

$$
\mathrm{K}_{\mathrm{a}}=\frac{\left[\mathrm{A}^{-}\right]\left[\mathrm{H}_{3} \mathrm{O}^{+}\right]}{[\mathrm{HA}]} \quad \mathrm{K}_{\mathrm{b}}=\frac{\left[\mathrm{BH}^{+}\right][-\mathrm{OH}]}{[\mathrm{B}]}
$$

$\left[\mathrm{A}^{-}\right]+[\mathrm{HA}]=\mathrm{c}_{\mathrm{a}}$ balance de masa $\left[\mathrm{BH}^{+}\right]+[\mathrm{B}]=\mathrm{c}_{\mathrm{b}}$

$\left[\mathrm{H}_{3} \mathrm{O}^{+}\right]=\left[\mathrm{A}^{-}\right]+[-\mathrm{OH}]$ balance de carga $[-\mathrm{OH}]=\left[\mathrm{BH}^{+}\right]+\left[\mathrm{H}_{3} \mathrm{O}^{+}\right]$ $\left[\mathrm{H}_{3} \mathrm{O}^{+}\right][-\mathrm{OH}\}=\mathrm{K}_{\mathrm{w}}$

$\left[\mathrm{H}_{3} \mathrm{O}^{+}\right\}\{-\mathrm{OH}]=\mathrm{K}_{w}$

La determinación de la concentración de $\mathrm{H}_{3} \mathrm{O}^{+}$o de ${ }^{-} \mathrm{OH}$ y del $\mathrm{pH}$ por estos sistemas de ecuaciones presupone la resolución de una ecuación đe tercer grado. Sin embargo, en muchos casos, es posible hacer aproximaciones que permitan facilitar los cálculos.

En relación con el uso de aproximaciones en los cálculos para los equilibrios ácido-base existen numerosos estudios de Narasaki $(1979,1980,1987)$.

Estas aproximaciones se efectúan sobre el balance de masa cuando el ácido o la base se pueden clasificar como fuertes o débiles y sobre el balance de carga cuando es posible despreciar la contribución del agua o la del ácido (o la de la base).

\section{1. ÁCIDO Y BASE FUERTES}

Un ácido y una base se definen como fuertes cuando $\mathrm{K}_{(\mathrm{a}} \mathrm{O}_{\mathrm{o})} / \mathrm{c}>18,05$; es decir, cuando ha reaccionado más del $95 \%$.

La aproximación sobre el balance de masa consiste, en estos casos, en despreciar las concentraciones, respectivamente, del ácido y de la base que no han reaccionado. Se tendrá, por consiguiente:

$$
\left[\mathrm{A}^{-}\right]=c_{a}(1) \quad\left[\mathrm{BH}^{+}\right]=\mathrm{c}_{\mathrm{b}}
$$

Los cálculos nos permitirán distinguir tres situaciones posibles:

1a. $\mathrm{c}_{\mathrm{a}(}\left(\mathrm{c}_{\mathrm{b})}>10^{-6.35}\right.$

Se puede despreciar la contribución a los $\mathrm{H}_{3} \mathrm{O}^{+}$y a los -OH debido a la disociación del agua. Por lo tanto:

$$
\left[\mathrm{H}_{3} \mathrm{O}^{+}\right]=\left[\mathrm{A}^{-}\right]=\mathrm{c}_{\mathrm{a}} \quad\left[{ }^{-} \mathrm{OH}\right]=\left[\mathrm{BH}^{+}\right]=\mathrm{c}_{\mathrm{b}}
$$

Ib. $c_{a t}\left(O C_{b)}<10^{-8,3}\right.$

Se puede despreciar la contribución a los $\mathrm{H}_{3} \mathrm{O}^{+}$y a los 
-OH debido a la reacción del ácido y de la base. Por lo tanto:

$$
\left[\mathrm{H}_{3} \mathrm{O}^{+}\right]=[-\mathrm{OH}]
$$

lc. $10^{-8,3} \leq \mathrm{c}_{\mathrm{a}}\left(o \mathrm{c}_{\mathrm{b}}\right) \leq 10^{-6,35}$

Ambas contribuciones deben ser consideradas ya que son comparables.

\section{2. ÁCIDO Y BASE DÉBILES}

Un ácido y una base se definen débiles cuanđo $\mathrm{K}_{(\mathrm{a}} \mathrm{O}_{\mathrm{b})} /$ $c<2,6 \times 10^{-3}$, es decir cuando reaccionan por debajo del 5\% (Narasaki 1979).

Se tendrá por consiguiente:

$$
[\mathrm{HA}]=\mathrm{c}_{\mathrm{a}}[\mathrm{B}]=\mathrm{c}_{\mathrm{b}}
$$

Los cálculos nos permiten distinguir tres situaciones posibles:

2a. $K_{a}, c_{a}\left(0 K_{b}, c_{b}\right)>2 \times 10^{-13}$

Se puede despreciar la contribución del agua; por lo que:

$$
\begin{array}{cc}
\mathrm{K}_{\mathrm{a}}=\left[\mathrm{H}_{3} \mathrm{O}^{+}\right]^{2} / \mathrm{c}_{\mathrm{a}} & \mathrm{K}_{\mathrm{b}}=\left[{ }^{-} \mathrm{OH}^{2}\right] / \mathrm{c}_{\mathrm{b}} \\
{\left[\mathrm{H}_{3} \mathrm{O}^{+}\right]=\sqrt{\mathrm{K}_{\mathrm{a}} \cdot \mathrm{c}_{\mathrm{a}}}} & {[-\mathrm{OH}]=\sqrt{\mathrm{K}_{\mathrm{b}} \cdot \mathrm{c}_{\mathrm{b}}}}
\end{array}
$$

2b. $K_{a} \cdot c_{a}\left(o K_{b} \cdot c_{b}\right)<5 \times 10^{-16}$

Se puede despreciar la contribución del ácido o de la base a $\left[\mathrm{H}_{3} \mathrm{O}^{+}\right]$y a $\mathrm{OH}^{-}$respectivamente, por lo que:

$$
\left[\mathrm{H}_{3} \mathrm{O}^{+}\right]=[-\mathrm{OH}]
$$

2c. $5 \times 10^{-16} \leq \mathrm{K}_{\mathrm{a}}, \mathrm{c}_{\mathrm{a}}\left(0 \mathrm{~K}_{\mathrm{b}}, \mathrm{c}_{\mathrm{b}}\right) \leq 2 \times 10^{-13}$

\section{NOTA}

Este artículo ha sido traducido del original italiano.

\section{REFERENCIAS BIBLIOGRÁFICAS}

FELTY, W.L., 1978. Journal Chemical Education, 55(9), p. 576.

NARASAKI, H., 1979. Talanta, 26, p. 605.

NARASAKI, H., 1980. Talanta, 27, p. 187.

NARASAKI, H., 1980. Talanta, 27, p. 193.

NARASAKI, H., 1980. Talanta, 27, p. 409.
Ambas contribuciones se deben contabilizar en el cálculo. Hay que aplicar las relaciones expresadas por las ecuaciones (Pauling 1976).

\section{3. ÁCIDO Y BASE "MEDIOS" O "INTER- MEDIOS}

Cuando $2,6 \times 10^{-13} \leq \mathrm{K}(\mathrm{a}$ o b $) / \mathrm{c} \leq 18,05$,

los ácidos y las bases no pueden ser clasificados ni entre los fuertes ni entre débiles. Los llamaremos "medios" o "intermedios".

En estos casos, en los balances de masa, no son lícitas las aproximaciones; por consiguiente:

$$
\left[\mathrm{A}^{-}\right]+[\mathrm{HA}]=\mathrm{c}_{\mathrm{a}} \quad[\mathrm{B}]+\left[\mathrm{BH}^{+}\right]=\mathrm{c}_{\mathrm{b}}
$$

Los cálculos no conducen, también en este caso, a tres posibles situaciones.

3a. $\left[\mathrm{H}_{3} \mathrm{O}^{+}\right]>4,47 \times 10^{-7}$

Se ha podido despreciar la contribución del agua y el resultado conseguido es correcto.

3b. $\left[\mathrm{H}_{3} \mathrm{O}^{+}\right]<2,23 \times 10^{-8}$

En este caso es despreciable la contribución a $\mathrm{H}_{3} \mathrm{O}^{+}$del ácido $\mathrm{y}$

$$
\left[\mathrm{H}_{3} \mathrm{O}^{+}\right]=[-\mathrm{OH}]=10^{-7}
$$

3c. $2,23 \times 10^{-8}<\left[\mathrm{H}_{3} \mathrm{O}^{+}\right]<4,47 \times 10^{-7}$

Ambas contribuciones deben ser consideradas, no puede conocerse el resultado; tenđrán que utilizarse las ecuaciones de tercer grado sin aproximaciones.

NARASAKI, H. y FRESENIUS Z., 1987. Anal. Chemical, 328, p. 633

PAULING, L., 1956. Journal Chemical Education, 53 (12), p. 828.

PAULING, L., 1976. Journal Chemical Education, 53 (12), p. 762 .

PAULING, L., The nature of the chemical bond, 3a. ed., pp. 618-621. (Comell University Press: Ithaca, New York). 